\title{
Surgical treatment of caseous mitral valve annulus calcification
}

\author{
Michael J. Davidson, MD, and Lawrence H. Cohn, MD, Boston, Mass
}

M itral annular calcification (MAC) is common in the elderly population. It can cause mitral regurgitation, mitral stenosis, and heart block and can complicate attempts to repair or replace the mitral valve. Extensive MAC can undergo liquefaction and simulate an intracardiac mass. Previously this rare entity was believed to be a benign incidental finding on imaging. We describe a case of caseous MAC mimicking a cardiac tumor and causing spontaneous cerebral embolization.

\section{Clinical Summary}

A 79-year-old woman was referred to our institution after experiencing painless monocular vision loss. Examination was consistent with central retinal artery embolism. Magnetic resonance imaging revealed bilateral microvascular infarctions in the anterior circulation. Transthoracic echocardiogram and a gated chest computed tomogram (Figure 1, A) showed a 3-cm heavily calcified mass associated with the posterior mitral annulus. Her presentation was initially believed to be most consistent with embolization from intracardiac tumor.

She was referred for operative intervention and underwent preoperative coronary angiography, which revealed significant singlevessel disease of the left anterior descending coronary artery. An intraoperative transesophageal echocardiogram again demonstrated a calcified cavitary mass associated with the posterior mitral annulus but only trace mitral regurgitation and no mitral stenosis (Figure 1, $B$ and $C$ ).

After initiation of cardiopulmonary bypass and left atriotomy, visual inspection did not reveal a mass. Instead, the posterior mitral valve leaflet appeared tented up; palpation of the posterior leaflet and lateral ventricular wall caused particulate fluid to express from under the leaflet. Needle aspiration produced more particulate, milky fluid; Gram stain demonstrated no organisms. The posterior mitral leaflet was then incised along the annulus, revealing a $3-\mathrm{cm}$ cavity filled with necrotic debris (Figure 2). The appearance was consistent with liquefaction necrosis of preexisting MAC. The interior of the cavity was debrided extensively, and the cavity appeared to extend into the lateral ventricular wall.

\footnotetext{
From the Division of Cardiac Surgery, Brigham and Women's Hospital, Boston, Mass.

Received for publication Nov 2, 2005; accepted for publication Nov 16, 2005.

Address for reprints: Lawrence H. Cohn, MD, Division of Cardiac Surgery, Brigham and Women's Hospital, 75 Francis St, Boston, MA 02115 (E-mail: Lcohn@partners.org).

J Thorac Cardiovasc Surg 2006;131:738-9

$0022-5223 / \$ 32.00$

Copyright @ 2006 by The American Association for Thoracic Surgery

doi:10.1016/j.jtcvs.2005.11.025
}

The incised leaflet edge was reattached to the valve annulus by using a running Prolene (Ethicon, Inc, Somerville, NJ) suture to seal the cavity. To obliterate the cavity completely, we performed a posterior leaflet-sparing mitral valve replacement, imbricating the posterior leaflet by using large noneverting annular sutures. A bioprosthetic valve (No. 25 Carpentier-Edwards pericardial valve; Edwards Lifesciences, Irvine, Calif) was chosen because the rigid valve strut would further compress the cavity to prevent reformation. Single-vessel coronary bypass was performed by using the left internal thoracic artery to the left anterior descending coronary artery, and the patient was weaned from cardiopulmonary bypass. A postoperative transesophageal echocardiogram showed effective obliteration of the cavity by the valve strut (Figure $1, D$ ).

Pathologic examination of the cavity contents revealed calcific degeneration without evidence of endocarditis. The patient had an uneventful postoperative recovery and was discharged home. Her vision loss persisted, but she had no further neurologic events during 6 months of follow-up.

\section{Discussion}

MAC is a common finding among the elderly and is largely asymptomatic, although it may be mistaken for intracardiac tumor. ${ }^{1}$ The entity described is a rare clinical finding, alternatively termed caseous calcification or liquefaction necrosis of MAC. The prevalence of caseous calcification was reported to be $2.7 \%$ of all cases of MAC in an autopsy series. ${ }^{2}$ A more recent study of 28,364 adults referred for echocardiography demonstrated MAC in 3007 $(10.6 \%)$ and caseous calcification in $19(0.63 \%$ of MAC cases and $0.067 \%$ of the population referred for echocardiography). ${ }^{3}$ Kronzon and colleagues ${ }^{4}$ found a similar prevalence of $0.055 \%$. Among the 19 cases examined by Harpaz and associates, ${ }^{3} 5(26 \%)$ had cerebrovascular events, although no causal link was established. Ultimately, only 1 patient in that series was referred for valve replacement because of concern for embolic potential, and 2 others underwent mitral valve replacement for valve dysfunction. No mass-related events were noted in the remaining 16 patients.

This patient demonstrates the embolic potential of caseous calcification of the mitral valve. Calcium deposits on MAC have previously been reported to cause cerebral emboli. ${ }^{5}$ Although the presence of asymptomatic caseous calcification might be followed up nonoperatively, any evidence of embolic phenomena should prompt operative intervention. Mitral replacement seems to be the most appropriate method to achieve cavity obliteration; there have been no reports of successful mitral valve preservation in this disease process. Indeed, the cavity is calcium lined and rigid and protrudes into the left ventricular wall. Overly aggressive debridement could risk perforation of the left ventricle, and simple unroofing of the cavity would continue to expose necrotic calcific debris to the systemic blood flow. A technique of noneverting annular sutures was useful both to avoid unnecessary tension in a calcified annulus and to assist in obliteration of the cavity. Posi- 

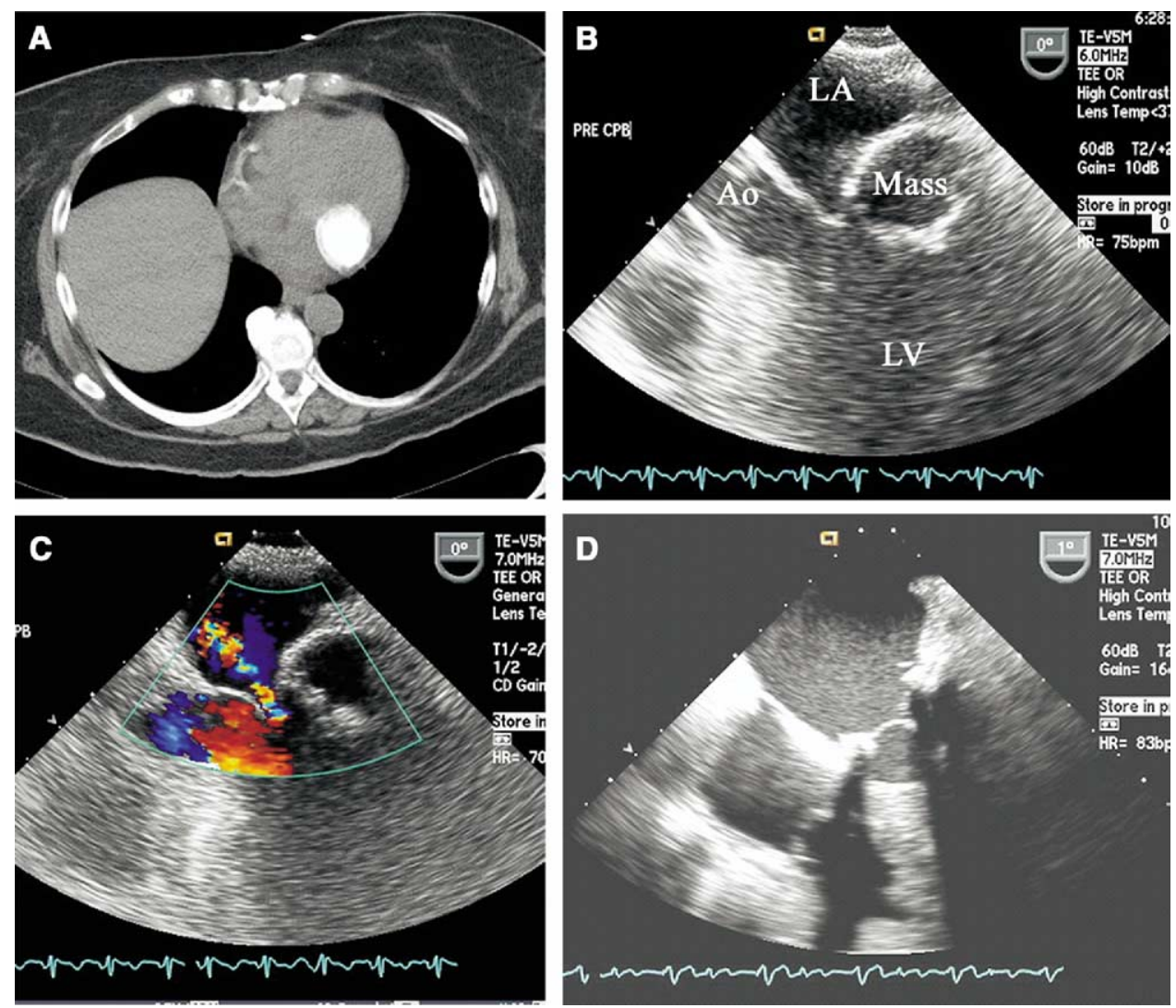

Figure 1. Appearance of the mitral annular mass on imaging. A, Computed tomographic scan showing a heavily calcified mass in the region of the posterior mitral valve. B, Intraoperative transesophageal echocardiogram (TEE) demonstrating a cavitary mass underlying the posterior leaflet of the mitral valve. C, Color Doppler image of systolic flow showing trace mitral regurgitation. D, Postoperative TEE shows a cavity obliterated by a bioprosthetic valve. $L A$, Left atrium; $L V$, left ventricle; $A o$, aorta.

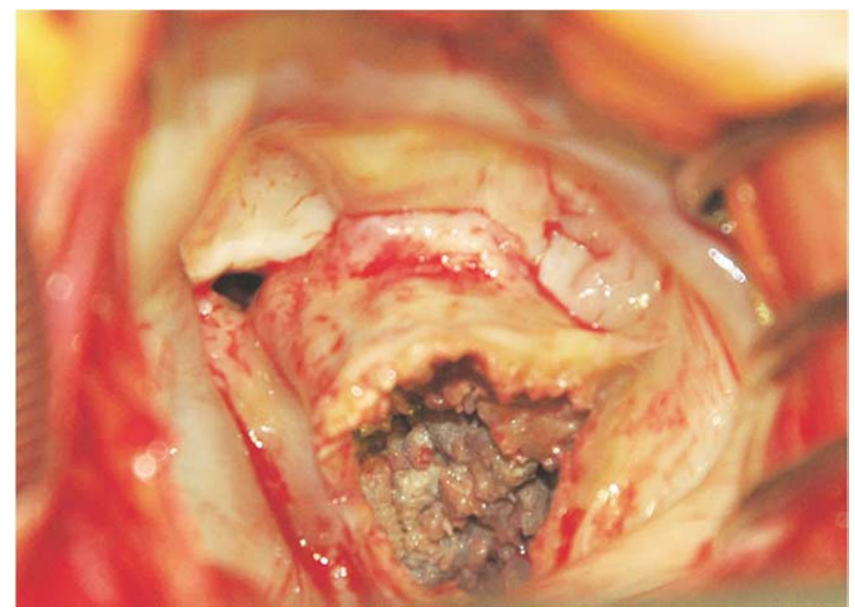

Figure 2. Interior of the cavity exposed after incision of the posterior leaflet along the mitral annulus, demonstrating necrotic debris within. tioning the bioprosthetic valve strut over the cavity seemed to provide additional structural reinforcement.

\section{References}

1. Teja K, Gibson RS, Nolan SP. Atrial extension of mitral annular calcification mimicking intracardiac tumor. Clin Cardiol. 1987;10:546-8.

2. Pomerance A. Pathological and clinical study of calcification of the mitral valve ring. J Clin Pathol. 1987;23:354-61.

3. Harpaz D, Auerbach I, Vered Z, Morro M, Tobar A, Rosenblatt S. Caseous calcification of the mitral annulus: a neglected, unrecognized diagnosis. J Am Soc Echocardiogr. 2001;14:825-31.

4. Kronzon I, Winer HE, Cohen ML. Sterile caseous mitral annular abscess. J Am Coll Cardiol. 1983;2:186-90.

5. Ridolfi RL, Hutchins GM. Spontaneous calcific emboli from calcific mitral annulus fibrosus. Arch Pathol Lab Med. 1976;100:117-20. 